\title{
On multigraphic and potentially multigraphic sequences
}

\author{
Dedicated to the memory of Antal Iványi
}

\author{
Shariefuddin PIRZADA \\ University of Kashmir \\ Department of Mathematics \\ Srinagar, Kashmir, India \\ email: \\ pirzadasd@kashmiruniversity.ac.in
}

\author{
Bilal Ahmad CHAT \\ University of Kashmir \\ Srinagar, Kashmir, India \\ email: bilalchat99@gmail.com
}

\author{
Uma Tul SAMEE \\ Islamia College for Science and Commerce, \\ Srinagar, Kashmir \\ email: pzsamee@yahoo.co.in
}

\begin{abstract}
An r-graph(or a multigraph) is a loopless graph in which no two vertices are joined by more than $r$ edges. An $r$-complete graph on $n$ vertices, denoted by $K_{n}^{(r)}$, is an r-graph on $n$ vertices in which each pair of vertices is joined by exactly $r$ edges. A non-increasing sequence $\pi=\left(d_{1}, d_{2}, \ldots, d_{n}\right)$ of non-negative integers is said to be $r$-graphic if it is realizable by an $r$-graph on $n$ vertices. An r-graphic sequence $\pi$ is said to be potentially $S_{L, M}^{(r)}$-graphic if it has a realization containing $S_{L, M}^{(r)}$ as a subgraph. We obtain conditions for an r-graphic sequence to be potentially $S_{L, M}^{(r)}$-graphic. These are generalizations from split graphs to $p$-tuple r-split graph.
\end{abstract}

Computing Classification System 1998: G.2.2

Mathematics Subject Classification 2010: 05C07

Key words and phrases: multigraph, multigraphic sequence, potentially multigraphic sequences, split graph 


\section{Introduction}

For a positive integer $r$, an $r$-graph(or multigraph) is a loopless graph in which no two vertices are joined by more than $r$ edges. An $r$-complete graph on $n$ vertices, denoted by $K_{n}^{(r)}$, is an r-graph on $n$ vertices in which each pair of vertices is joined by exactly $r$ edges. Clearly, $K_{n}^{(1)}=K_{n}$. A non-increasing sequence $\pi=\left(d_{1}, d_{2}, \ldots, d_{n}\right)$ of non-negative integers is said to be $r$-graphic if it is the degree sequence of an $r$-graph $G$ on $n$ vertices, and such an $r$-graph $G$ is referred to as a realization of $\pi$. We take $\sigma(\pi)=\sum_{i=1}^{n} d_{i}$. For graph theoretical notations and definitions we refer to [9].

Let $\pi=\left(d_{1}, d_{2}, \ldots, d_{n}\right)$ be a non-increasing sequence of non-negative integers with $d_{1} \leq \sum_{i=2}^{n} \min \left\{r, d_{i}\right\}$. Define $\pi_{k}^{\prime}=\left(d_{1}^{\prime}, d_{2}^{\prime}, \ldots, d_{n-1}^{\prime}\right)$ to be the nonincreasing rearrangement of the sequence obtained from

$$
\left(d_{1}, d_{2}, \ldots, d_{k-1}, d_{k+1}, \ldots, d_{n}\right)
$$

by reducing by 1 the remaining largest terms that have not been reduced $r$ times, and repeating the procedure $d_{k}$ times. $\pi_{k}^{\prime}$ is called the residual sequence obtained from $\pi$ by laying off $d_{k}$.

The following three results due to Chungphaisian [2] are generalizations from 1-graphs to r-graphs of three well-known results, one by Erdős and Gallai [3], one by Kleitman and Wang [6] and one by Fulkerson, Hoffman and Mcandrew [5].

Theorem 1 [2] Let $\pi=\left(\mathrm{d}_{1}, \mathrm{~d}_{2}, \ldots, \mathrm{d}_{\mathrm{n}}\right)$ be a non-increasing sequence of nonnegative integers, where $\sigma(\pi)$ is even. Then $\pi$ is $r$-graphic if and only if for each positive integer $\mathrm{t} \leq \mathrm{n}$,

$$
\sum_{i=1}^{t} d_{i} \leq r t(t-1)+\sum_{i=t+1}^{n} \min \left\{r t, d_{i}\right\} .
$$

Theorem 2 [2] $\pi$ is r-graphic if and only if $\pi_{\mathrm{k}}^{\prime}$ is $\mathrm{r}$-graphic.

Let the subgraph $\mathrm{H}$ on the vertices $v_{i}, v_{j}, v_{k}, v_{l}$ of a multigraph $\mathrm{G}$ contain the edges $v_{i} v_{j}$ and $v_{k} v_{l}$. The operation of deleting these edges and introducing a pair of new edges $v_{i} v_{l}$ and $v_{j} v_{k}$, or $v_{i} v_{k}$ and $v_{j} v_{l}$ is called an elementary degree preserving transformation. If this operation is performed $r$ times on the same edge set, it is called $r$-exchange. 
Theorem 3 [2] Let $\pi$ be an $\mathrm{r}$-graphic sequence, and let $\mathrm{G}$ and $\mathrm{G}^{\prime}$ be realizations of $\pi$. Then there is a sequence of $r$-exchanges, $\mathrm{E}_{1}, \ldots, \mathrm{E}_{\mathrm{k}}$ such that the application of these $\mathrm{r}$-exchanges to $\mathrm{G}$ in order will result in $\mathrm{G}^{\prime}$.

An r-graphic sequence $\pi$ is said to be potentially $K_{m+1}^{(r)}$ if there exists a realization of $\pi$ containing $K_{m+1}^{(r)}$ as a subgraph. If $\pi$ has a realization $G$ containing $K_{m+1}^{(r)}$ on the $m+1$ vertices of highest degree in $G$, then $\pi$ is said to be potentially $A_{m+1}^{(r)}$-graphic. As a special case of Lemma 2.1 in [13], Yin showed that an $r$-graphic sequence is potentially $K_{m+1}^{(r)}$-graphic if and only if it is potentially $A_{m+1}^{(r)}$-graphic.

The $r$-join (complete product) of two $r$-graphs $G_{1}$ and $G_{2}$ is a graph $G=$ $G_{1} \vee G_{2}$ with vertex set $V\left(G_{1}\right) \cup V\left(G_{2}\right)$ and the edge set consisting of all edges of $G_{1}$ and $G_{2}$ together with the edges joining each vertex of $G_{1}$ with every vertex of $G_{2}$ by exactly $r$ edges. Let $K_{l}^{(r)}$ and $K_{m}^{(r)}$ be complete $r$-graphs with $l$ and $m$ vertices respectively, that is the complete graphs having exactly $r$ edges between every two vertices. The r-split graph of $\mathrm{K}_{\mathrm{l}}^{(\mathrm{r})}$ and $\overline{\mathrm{K}}_{\mathrm{m}}^{(\mathrm{r})}$ denoted by $\bar{S}_{l, m}^{(r)}$ is the graph $K_{l}^{(r)} \vee \overline{K_{m}^{(r)}}$ having $l+m$ vertices, where $\overline{K_{m}^{(r)}}$ (having no edges) is the complement of $K_{m}^{(r)}$. [14]. If $\pi$ has a realization $G$ containing $\bar{S}_{l, m}$ on the $l+m$ vertices of highest degree in $G$, then $\pi$ is said to be potentially $\overline{\mathrm{A}}_{l, \mathrm{~m} \text {-graphic. }}$

The following two results due to Yin [13] are generalizations from 1-graphs to r-graphs of two well-known results given by A. R. Rao [12].

Theorem 4 [13] Let $\mathrm{n} \geq l+1$ and $\pi=\left(\mathrm{d}_{1}, \mathrm{~d}_{2}, \ldots, \mathrm{d}_{\mathrm{n}}\right)$ be an $\mathrm{r}$-graphic sequence with $\mathrm{d}_{l+1} \geq \mathrm{rl}$. Then $\pi$ is potentially $\mathrm{A}_{l+1}^{(\mathrm{r})}$-graphic if and only if $\pi_{l+1}$ is r-graphic.

Theorem 5 [13] Let $\mathrm{n} \geq \mathrm{l}+1$ and $\pi=\left(\mathrm{d}_{1}, \mathrm{~d}_{2}, \ldots, \mathrm{d}_{\mathrm{n}}\right)$ be an $\mathrm{r}$-graphic sequence with $\mathrm{d}_{\mathrm{l}+1} \geq 2 \mathrm{rl}-1$, then $\pi$ is potentially $\mathrm{K}_{\mathrm{l}+1}^{(\mathrm{r})}$.

An extremal problem for 1-graphic sequences to be potentially $K_{l}^{(1)}$-graphic was considered by Erdős, Jacobson and Lehel [4] and solved by Li et al. [7, 8]. Yin [13] generalized this extremal problem and the Erdős-Jacobson-Lehel conjecture from 1-graphs to $r$-graphs.

In 2014, the authors [10] proved the following assertion. 
Theorem 6 [10] If $\mathrm{G}_{1}$ is a realization of $\pi_{1}=\left(\mathrm{d}_{1}^{1}, \ldots, \mathrm{d}_{\mathrm{m}}^{1}\right)$ containing $\mathrm{K}_{\mathrm{p}}$ as a subgraph and $\mathrm{G}_{2}$ is a realization of $\pi_{2}=\left(\mathrm{d}_{1}^{2}, \ldots, \mathrm{d}_{\mathrm{n}}^{2}\right)$ containing $\mathrm{K}_{\mathrm{q}}$ as a subgraph, then the degree sequence $\pi=\left(\mathrm{d}_{1}, \ldots, \mathrm{d}_{\mathrm{m}+\mathrm{n}}\right)$ of the join of $\mathrm{G}_{1}$ and $\mathrm{G}_{2}$ is potentially $\mathrm{K}_{\mathrm{p}+\mathrm{q}}$-graphic.

The following two results for simple graphs are due to Yin [14].

Theorem 7 [14] $\pi$ is potentially $\bar{A}_{l, m}$-graphic if and only if $\pi_{l}$ is graphic.

Theorem 8 [14] Let $\mathrm{n} \geq \mathrm{l}+\mathrm{m}$ and let $\pi=\left(\mathrm{d}_{1}, \mathrm{~d}_{2}, \cdots, \mathrm{d}_{\mathrm{n}}\right)$ be a nonincreasing graphic sequence. If $\mathrm{d}_{\mathrm{l}+\mathrm{m}} \geq 2 \mathrm{l}+\mathrm{m}-2$, then $\pi$ is potentially $\overline{\mathrm{A}}_{\mathrm{l}, \mathrm{m}}$ graphic.

A condition for a graphic sequence $\pi$ to be potentially $\mathrm{K}_{4}-\mathrm{e}$ graphic can be found in [11], where $K_{4}-e$ is the graph obtained from the complete graph $\mathrm{K}_{4}$ by deleting one edge $e$.

\section{Bounds on the sum of squares of degrees of a multigraph}

From the Cauchy-Schwarz inequality, we have

$$
\sum_{i=1}^{n}\left|a_{i} b_{i}\right| \leq\left(\sum_{i=1}^{n}\left|a_{i}\right|^{2}\right)^{\frac{1}{2}}\left(\sum_{i=1}^{n}\left|b_{i}\right|^{2}\right)^{\frac{1}{2}},
$$

Taking $a_{i}=d_{i}$ and $b_{i}=1$, we have $\left(\sum_{i=1}^{n} d_{i}\right)^{2} \leq n \sum_{i=1}^{n} d_{i}^{2}$ which implies $\frac{1}{n}\left(\sum_{i=1}^{n} d_{i}\right)^{2} \leq\left(\sum_{i=1}^{n} d_{i}^{2}\right)$. From this and the hand shaking Lemma $\sum_{i=1}^{n} d_{i}=2|E|$, we have $\frac{4|E|^{2}}{n}=\frac{1}{n}\left(\sum_{i=1}^{n} d_{i}\right)^{2} \leq \sum_{i=1}^{n} d_{i}^{2}$.

Now we have the following observation, the proof is by using the same argument as in Theorem 1 of [1].

Lemma 9 For an $r$-graph $\mathrm{G}, \sum_{i=1}^{n} \mathrm{~d}_{i}^{2} \leq|\mathrm{E}|\left(\mathrm{r}(\mathrm{n}-2)+\frac{2|\mathrm{E}|}{\mathrm{n}-1}\right)$. 


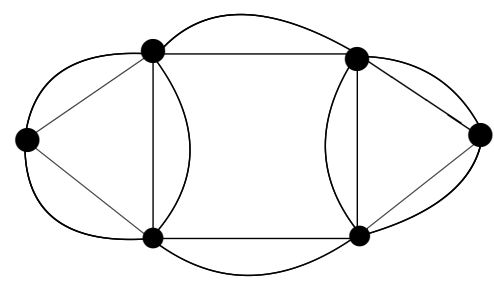

Figure 1: A 2-graph

Remark 10 From Lemma 9, we observe that

$$
\frac{4|E|^{2}}{n} \leq \sum_{i=1}^{n} d_{i}^{2} \leq|E|\left(r(n-2)+\frac{2|E|}{n-1}\right) .
$$

The following example shows that the equality does not hold in the above inequality.

Example 11 Consider the 2-graph as shown in Figure 1.

Here, $\frac{4|\mathrm{E}|^{2}}{n}=\frac{4 \times 16^{2}}{6}=\frac{512}{3}<4^{2}+6^{2}+6^{2}+6^{2}+6^{2}+4^{2}=176<16(2(6-2)+$ $\left.\frac{2 \times 16}{6-1}\right)=\frac{1152}{5}$.

Now, we have the following result.

Lemma 12 A multigraph $\mathrm{G}$ is regular if and only if $\frac{4|\mathrm{E}|^{2}}{n}=\sum_{i=1}^{n} \mathrm{~d}_{i}^{2}$.

Proof. Suppose an $r$-graph $G$ is regular of degree $b$. Then $2|E|=n b$ and $d_{i}=b$ for all $i=1,2, \ldots, n$. We know that $\sum_{i=1}^{n} d_{i}^{2}=n b^{2}$ and $\frac{4|E|^{2}}{n}=\frac{1}{n} 4 \frac{1}{4} n^{2} b^{2}=n b^{2}$. These together give $\sum_{i=1}^{n} d_{i}^{2}=\frac{4|E|^{2}}{n}$.

Conversely, suppose that $\sum_{i=1}^{n} d_{i}^{2}=\frac{4|E|^{2}}{n}$. Then $\frac{4}{n}|E|^{2}=\sum_{i=1}^{n} d_{i}^{2}$. This implies that $\frac{1}{n}\left(d_{1}^{2}+d_{2}^{2}+\ldots+d_{n}^{2}+2\left(d_{1} d_{2}+d_{1} d_{3}+\ldots+d_{1} d_{n}\right)+\ldots+2\left(d_{n-2} d_{n-1}+\right.\right.$ $\left.\left.d_{n-2} d_{n}\right)+2\left(d_{n-1} d_{n}\right)\right)-\left(d_{1}^{2}+d_{2}^{2}+\ldots+d_{n}^{2}\right)=0$, which on simplification gives 
$\frac{1}{n}\left(\left(d_{1}-d_{2}\right)^{2}+\left(d_{1}-d_{3}\right)^{2}+\ldots+\left(d_{1}-d_{n}\right)^{2}+\left(d_{2}-d_{3}\right)^{2}+\left(d_{2}-d_{4}\right)^{2}+\ldots+\right.$ $\left.\left(d_{2}-d_{n}\right)^{2}+\ldots+\left(d_{n-1}-d_{n}\right)^{2}\right)=0$. From this, we see that each term on left side is non-negative for every $i, j$ and right side is equal to zero. Therefore the above equation is possible when $d_{i}=d_{j}$ for every $i, j=1,2, \ldots, n$ and hence $\mathrm{G}$ is a regular graph.

Now, we have the following observation.

Lemma 13 Let $\mathrm{G}$ be an $\mathrm{r}$-graph with $\mathrm{n}>2$ vertices. Then $\mathrm{G}$ is a complete graph $\mathrm{K}_{\mathrm{n}}^{\mathrm{r}}$ if and only if $\frac{4|\mathrm{E}|^{2}}{\mathrm{n}}=\sum_{i=1}^{n} \mathrm{~d}_{i}^{2}=|\mathrm{E}|\left(\mathrm{r}(\mathrm{n}-2)+\frac{2|\mathrm{E}|}{\mathrm{n}-1}\right)$.

Proof. First we note that an r-graph G is a complete r-graph if and only if $|E|=\frac{1}{2} r n(n-1)$. Moreover, we know that $|E|=\frac{1}{2} n r(n-1)$, which implies that $2|E|(n-2)+2|E| n=n r(n-1)(n-2)+2|E| n$ and on simplication gives $\frac{4|E|^{2}}{n}=|E|\left(r(n-2)+\frac{2|E|}{n-1}\right)$. Thus the result follows.

The following result partially answers the question raised in Remark 10.

Theorem 14 A bipartite multigraph $\mathrm{G}=\mathrm{K}_{\mathrm{l}, \mathrm{m}}^{(\mathrm{r})}$, where $\mathrm{m}>1$, is an $\mathrm{r}$-star graph $\mathrm{K}_{1, n-1}^{(\mathrm{r})}$ if and only if $\sum_{i=1}^{\mathrm{n}} \mathrm{d}_{\mathrm{i}}^{2}=|\mathrm{E}|\left(\mathrm{r}(\mathrm{n}-2)+\frac{2|\mathrm{E}|}{\mathrm{n}-1}\right)$.

Proof. Let $K_{l, m}^{(r)}$ be an $r$-complete bipartite graph, where $m>1, n=l+m$ and $|\mathrm{E}|=\mathrm{rlm}$. There are $l$-vertices each of whose degree is $r \times m$ and $m$ vertices each of whose degree is $r \times l$, so $\sum_{i=1}^{n} d_{i}^{2}=l(r m)^{2}+m(r l)^{2}=l r^{2} m^{2}+m r^{2} l^{2}=$ $r^{2}\left(\mathrm{~lm}^{2}+m l^{2}\right)=r^{2} \operatorname{lm}(l+m)$. Therefore, we have $|E|\left(r(n-2)+\frac{2|E|}{n-1}\right)=$ $r l m\left(r(l+m-2)+\frac{2 r l m}{n-1}\right)=r^{2} l m\left(\frac{l^{2}+m^{2}+4 l m-3 l-3 m+2}{l+m-1}\right)$. Therefore, $r^{2} \operatorname{lm}(l+m)=$ $r^{2} \operatorname{lm}\left(\frac{l^{2}+m^{2}+4 l m-3 l-3 m+2}{l+m-1}\right)$, which gives $l=1$. Hence the result follows.

\section{$3 \quad$ Potentially r-graphic sequences}

Definition 15 Let $\overline{\mathrm{S}}_{\mathrm{r}_{1}, \mathrm{~s}_{1}}^{(\mathrm{r})}, \overline{\mathrm{S}}_{\mathrm{r}_{2}, \mathrm{~s}_{2}}^{(\mathrm{r})}, \ldots, \overline{\mathrm{S}}_{\mathrm{r}_{\mathrm{p}}, \mathrm{s}_{\mathrm{p}}}^{(\mathrm{r})}$ be $\mathrm{r}$-split graphs, respectively with $r_{1}+s_{1}, r_{2}+s_{2}, \ldots, r_{p}+s_{p}$ vertices. Let $L=\sum_{i=1}^{p} r_{i}$ and $M=\sum_{i=1}^{p} s_{i}$. Then 
the $\mathrm{p}$-tuple $\mathrm{r}$-split graph, denoted by $\mathrm{S}_{\mathrm{L}, \mathrm{M}}^{(\mathrm{r})}$, is the graph

$$
S_{L, M}^{(r)}=S_{\sum_{i=1}^{p} r_{i}, \sum_{i=1}^{p} s_{i}}^{(r)}=\bar{S}_{r_{1}, s_{1}}^{(r)} \vee \bar{S}_{r_{2}, s_{2}}^{(r)} \vee \ldots \vee \bar{S}_{r_{p}, s_{p}}^{(r)}
$$

Clearly $S_{L, M}^{r}$ has vertex set $\bigcup_{i=1}^{p} V\left(\bar{S}_{r_{i}, s_{i}}^{(r)}\right)$ and the edge set consists of all edges of $\bar{S}_{r_{1}, s_{1}}^{(r)}, \bar{S}_{r_{2}, s_{2}}^{(r)}, \ldots, \bar{S}_{r_{p}, s_{p}}^{(r)}$ together with the edges joining each vertex of $\bar{S}_{r_{i}, s_{i}}^{(r)}$ with every vertex of $\bar{S}_{r_{j}, s_{j}}^{(r)}$ by exactly r-edges for every $i, j$ with $i \neq j$.

An r-graphic sequence $\pi$ is said to be potentially $S_{L+M}^{(r)}$-graphic if there exists a realization of $\pi$ containing $S_{L+M}^{(r)}$ as a subgraph. If $\pi$ has a realization $G$ containing $S_{\mathrm{L}+\mathrm{M}}^{(\mathrm{r})}$ on the $\mathrm{L}+\mathrm{M}$ vertices of highest degree in $\mathrm{G}$, then $\pi$ is said to be potentially $A_{L+M}^{(r)}$-graphic.

Let $n \geq L+M$ and let $\pi=\left(d_{1}, \ldots, d_{n}\right)$ be a non-increasing sequence of non-negative integers with $d_{L} \geq r(L+M)-1$ and $d_{L+M} \geq r L$. We define sequences $\pi_{1}, \ldots, \pi_{\mathrm{L}}$ as follows. Construct the sequence

$$
\pi_{1}=\left(d_{2}-r, \ldots, d_{L}-r, d_{L+1}-r, \ldots, d_{L+M}-r, d_{L+M+1}^{1}, \ldots, d_{n}^{1}\right)
$$

from $\pi$ by reducing 1 from the largest term that have not been already reduced $r$ times, and then reordering the last $n-L-M$ terms to be non-increasing. For $2 \leq i \leq r$, construct

$$
\pi_{i}=\left(d_{i+1}-i r, \ldots, d_{L}-i r, d_{L+1}-i r, \ldots, d_{L+M}-i r, d_{L+M+1}^{i}, \ldots, d_{n}^{i}\right)
$$

from

$$
\begin{aligned}
\pi_{i-1}= & \left(d_{i}-(i-1) r, \ldots, d_{L}-(i-1) r, d_{L+1}-(i-1) r, \ldots,\right. \\
& \left.d_{L+M}-(i-1) r, d_{L+M+1}^{i-1}, \ldots, d_{n}^{i-1}\right)
\end{aligned}
$$

by deleting $d_{i}-(i-1) r$, reducing the first $d_{i}-(i-1) r$ remaining terms of $d_{i-1}$ by one that have not been already reduced $r$ times, and then reordering the last $n-L-M$ terms to be non-increasing.

We start with the following lemma. 
Lemma 16 If $\pi=\left(\mathrm{d}_{1}, \mathrm{~d}_{2}, \ldots, \mathrm{d}_{\mathrm{n}}\right)$ is the graphic sequence of $\mathrm{S}_{\mathrm{L}, \mathrm{M}}^{(\mathrm{r})}$, then $\pi=\left(\left(\sum_{i=1}^{m} r\left(r_{i}+s_{i}-1\right)\right)^{r_{j}},\left(\sum_{i=1}^{m} r r_{i}+\sum_{i=1, i \neq j}^{m} r s_{i}\right)^{s_{j}}\right)$, for $j=1,2, \ldots, m$.

Proof. To prove the result we use induction on $\mathrm{m}$.

For $\mathrm{m}=1$, the result is obviously true. For $\mathrm{m}=2$, we have $\mathrm{S}_{\sum_{i=1}^{2} r_{i}, \sum_{i=1}^{2} s_{i}}$ Therefore for every $i=1,2, \ldots, r_{1}$ and $i=1,2,3, \ldots=r_{2}$ and $j=$ $1,2,3, \ldots, s_{1}$ and $j=1,2,3, \ldots, s_{2}$

$$
\overline{d_{i}}=d_{i}+r\left(r_{2}+s_{2}\right)
$$

and

$$
\overline{d_{j}}=r\left(r_{1}+r_{2}+s_{2}\right),
$$

where $\overline{d_{i}}$ and $\overline{d_{j}}$ are respectively the degree of $\overline{v_{i}^{\text {th }}}$ and $\overline{v_{j}^{\text {th }}}$ vertex in $S_{r_{1}+r_{2}, s_{1}+s_{2}}$ and $d_{i}$ is the degree of $i^{\text {th }}$ vertex in $K_{r_{1}}$. Equations (1) and (2) hold for every $\mathrm{i}, \mathrm{j}$. Thus the graphic sequence $\pi^{2}$ of $S_{\mathrm{r}_{1}+r_{2}, s_{1}+s_{2}}$ is

$$
\begin{aligned}
\pi^{2} & =\left(\left(r\left(r_{1}+s_{1}-1\right)+r\left(r_{2}+s_{2}\right)^{r_{1}},\left(r\left(r_{1}+s_{1}-1\right)+r\left(r_{2}+s_{2}\right)^{r_{2}},\right.\right.\right. \\
& \left.\left(r\left(r_{1}+r_{2}+s_{2}\right)\right)^{s_{1}},\left(r\left(r_{1}+r_{2}+s_{2}\right)\right)^{s_{2}}\right) \\
& =\left(\left(\sum_{i=1}^{2} r\left(r_{i}+s_{i}-1\right)\right)^{r_{j}},\left(\sum_{i=1}^{m} r r_{i}+\sum_{i=1, i \neq j}^{m} r s_{i}\right)^{r_{j}}\right), \text { for } j=1,2 .
\end{aligned}
$$

This shows that the result is true for $m=2$. Assume that the result holds for $\mathrm{m}=\mathrm{k}-1$, therefore for all $\mathrm{j}=1,2, \cdots, \mathrm{k}-1$,

$$
\pi^{k-1}=\left(\left(\sum_{i=1}^{k-1} r\left(r_{i}+s_{i}-1\right)\right)^{r_{j}},\left(\sum_{i=1}^{k-1} r r_{i}+\sum_{i=1, i \neq j}^{k-1} r s_{i}\right)^{r_{j}}\right), \text { for } j=1,2 .
$$

Now for $m=k$,

$$
\begin{aligned}
\mathrm{G} & =\mathrm{S}_{\mathrm{r}_{1}, \mathrm{~s}_{1}}^{(\mathrm{r})} \vee \mathrm{S}_{\mathrm{r}_{2}, \mathrm{~s}_{2}}^{(\mathrm{r})} \vee \ldots \vee S_{\mathrm{r}_{k-1}, s_{k-1}}^{(\mathrm{r})} \vee S_{\mathrm{r}_{k}, s_{k}}^{(\mathrm{r})} \\
& =A \vee S_{r_{k}, s_{k}}^{(\mathrm{r})}, \quad \text { where } \quad A=S_{\mathrm{r}_{1}, s_{1}}^{(\mathrm{r})} \vee S_{\mathrm{r}_{2}, s_{2}}^{(\mathrm{r})} \vee \ldots \vee S_{r_{k-1}, s_{k-1}}^{(\mathrm{r})} .
\end{aligned}
$$


Since the result is proved for all $m=k-1$ and using the fact that the result is proved for each pair and since the result is already proved for $k=2$, it follows by induction hypothesis that result holds for $m=k$ also. That is,

$$
\pi=\left(\left(\sum_{i=1}^{k} r\left(r_{i}+s_{i}-1\right)\right)^{r_{j}},\left(\sum_{i=1}^{k} r r_{i}+\sum_{i=1, i \neq j}^{k} r s_{i}\right)^{s_{j}}\right), \text { for } j=1,2, \ldots, k
$$

This proves the lemma.

Lemma 17 A non-increasing integer sequence $\pi=\left(\mathrm{d}_{1}, \ldots, \mathrm{d}_{\mathfrak{n}}\right)$ is potentially $\mathrm{A}_{\mathrm{L}, \mathrm{M}^{-}}^{(\mathrm{r})}$ graphic if and only if it is potentially $\mathrm{S}_{\mathrm{L}, \mathrm{M}^{-}}^{(\mathrm{r})}$ graphic.

Proof. We only need to prove that if $\pi=\left(d_{1}, \ldots, d_{n}\right)$ is potentially $S_{L, M^{-}}^{(r)}$ graphic, then it is potentially $A_{L, M}^{(r)}$-graphic. We choose a realization $G$ of $\pi$ with vertex set $\mathrm{V}(\mathrm{G})=\left\{v_{1}, \ldots, v_{n}\right\}$ such that $\mathrm{d}_{\mathrm{G}}\left(v_{\mathrm{i}}\right)=\mathrm{d}_{\mathrm{i}}$ for $1 \leq i \leq$ $\mathrm{n}$, the induced $\mathrm{r}$-subgraph $\mathrm{G}\left[\left\{v_{1}, \ldots, v_{\mathrm{L}+\mathrm{M}}\right\}\right]$ of $\left\{v_{1}, \ldots, v_{\mathrm{L}+\mathrm{M}}\right\}$ in $\mathrm{G}$ contains $S_{\mathrm{L}, M}^{(\mathrm{r})}$ as its r-subgraph and $\left|\mathrm{V}\left(\mathrm{K}_{\mathrm{L}}^{(\mathrm{r})}\right) \cap\left\{v_{1}, \ldots, v_{\mathrm{L}}\right\}\right|$ is maximum. Denote $\mathrm{H}=$ $\mathrm{G}\left[\left\{v_{1}, \ldots, v_{\mathrm{L}+\mathrm{M}}\right\}\right]$. If $\left|\mathrm{V}\left(\mathrm{K}_{\mathrm{L}}^{(\mathrm{r})}\right) \cap\left\{v_{1}, \ldots, \mathrm{d}_{\mathrm{L}}\right\}\right|=\mathrm{L}$, that is, $\mathrm{V}\left(\mathrm{K}_{\mathrm{L}}^{(\mathrm{r})}\right)=\left\{v_{1}, \ldots, v_{\mathrm{L}}\right\}$,

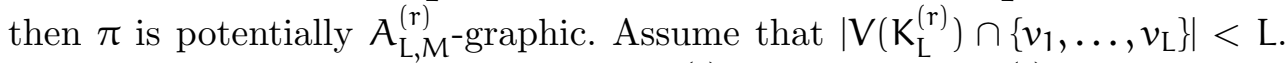
Then there exists $v_{i} \in\left\{v_{1}, \ldots, v_{\mathrm{L}}\right\} \backslash \mathrm{V}\left(\mathrm{K}_{\mathrm{L}}^{(\mathrm{r})}\right)$ and a $v_{\mathrm{j}} \in \mathrm{V}\left(\mathrm{K}_{\mathrm{L}}^{(\mathrm{r})}\right) \backslash\left\{v_{1}, \ldots, v_{\mathrm{L}}\right\}$. Let $\mathrm{A}=\mathrm{N}_{\mathrm{H}}\left(v_{\mathrm{j}}\right) \backslash\left(\left\{v_{i}\right\} \cup \mathrm{N}_{\mathrm{H}}\left(v_{\mathrm{i}}\right)\right)$ and $\mathrm{B}=\mathrm{N}_{\mathrm{G}}\left(v_{\mathrm{i}}\right) \backslash\left(\left\{v_{\mathrm{j}}\right\} \cup \mathrm{N}_{\mathrm{G}}\left(v_{\mathrm{j}}\right)\right)$. Since $\mathrm{d}_{\mathrm{G}}\left(v_{\mathrm{i}}\right) \geq \mathrm{d}_{\mathrm{G}}\left(v_{\mathrm{j}}\right)$, we have $|\mathrm{B}| \geq|\mathrm{A}|$. Let $\mathrm{C}$ be any subset of $\mathrm{B}$ such that $|C|=|A|$. Now form a new realization $G^{\prime}$ of $\pi$ by a sequence of $r$-exchanges to the r-edges of the star centralized at $v_{j}$ with end vertices in $A$ with the non $r$-edges of the star centralized at $v_{j}$ with end vertices in $C$, and by a sequence of $r$-exchange the $r$-edges of the star centralized at $v_{i}$ with end vertices in $C$ with the non $r$-edges of the star centralized at $v_{i}$ with end vertices in $A$. It is easy to see that $\mathrm{G}^{\prime}$ contains $\mathrm{S}_{\mathrm{L}, \mathrm{M}}^{(\mathrm{r})}$ on $\left\{v_{1}, \ldots v_{\mathrm{L}+\mathrm{M}}\right\}$ so that $\left|\mathrm{V}\left(\mathrm{K}_{\mathrm{L}}^{(\mathrm{r})}\right) \cap\left\{v_{1}, \ldots, v_{\mathrm{L}}\right\}\right|$ is larger than that of $\mathrm{G}$, which contradicts to the choice of $\mathrm{G}$.

We use the Havel-Hakimi procedure to test whether or not an r-graphic sequence $\pi$ is potentially $A_{L, M}^{(r)}$-graphic.

Theorem 18 For $\mathrm{r} \geq 1$ and $\mathrm{n} \geq 1$, an $\mathrm{r}$-graphic sequence $\pi=\left(\mathrm{d}_{1}, \ldots, \mathrm{d}_{\mathrm{n}}\right)$ is potentially $\mathrm{A}_{\mathrm{L}, \mathrm{M}}^{(\mathrm{r})}$-graphic if and only if $\pi_{\mathrm{L}}$ is $\mathrm{r}$-graphic.

Proof. Assume that $\pi$ is potentially $A_{L, M}^{(r)}$-graphic. Then $\pi$ has a realization $G$ with the vertex set $V(G)=\left\{v_{1}, \ldots, v_{n}\right\}$ such that $d_{G}\left(v_{i}\right)=d_{i}$ for $(1 \leq i \leq n)$ 
and $G$ contains $S_{L, M}^{(r)}$ on the vertices $v_{1}, \ldots, v_{L+M}$, where $L+M \leq n$, so that $\mathrm{V}^{(\mathrm{r})}\left(\mathrm{K}_{\mathrm{L}}\right)=\left\{v_{1}, \ldots, v_{\mathrm{L}}\right\}$ and $\mathrm{V}\left(\overline{\mathrm{K}}_{\mathrm{M}}^{(\mathrm{r})}\right)=\left\{v_{\mathrm{L}+1}, \ldots, v_{\mathrm{L}+\mathrm{M}}\right\}$. By applying a sequence of $r$-exchanges to $G$ in order we will show that there is one such realization $\mathrm{G}^{\prime}$ such that $\mathrm{G}^{\prime} \backslash v_{1}$ has degree sequence $\pi_{1}$. If not, we may choose such a realization $\mathrm{H}$ of $\mathrm{r}$-graphic sequence $\pi$ such that the number of vertices adjacent to $v_{1}$ in $\left\{v_{\mathrm{L}+\mathrm{M}+1}, \ldots, v_{\mathrm{d}_{1}+1}\right\}$ is maximum. Let $v_{\mathrm{i}} \in\left\{v_{\mathrm{L}+\mathrm{M}+1}, \ldots, v_{\mathrm{d}_{1}+1}\right\}$ and assume that there is no edge between $v_{1}$ and $v_{i}$ and let $v_{j} \in\left\{v_{\mathrm{d}_{1}+2}, \ldots, v_{n}\right\}$ and there are $r$ edges between $v_{1}$ and $v_{j}$. We may assume that $d_{i}>d_{j}$. Hence there is a vertex $v_{t}, t \neq i, j$ such that there are $r$ edges between $v_{i}$ and $v_{t}$ and no edge between $v_{j}$ and $v_{\mathrm{t}}$. Clearly $\mathrm{G}=\left(\mathrm{H} \backslash\left\{v_{1}^{(\mathrm{r})} v_{j}, v_{i}^{(\mathrm{r})} v_{\mathrm{t}}\right\}\right) \bigcup\left\{v_{1}^{(\mathrm{r})} v_{i}, v_{j}^{(\mathrm{r})} v_{\mathrm{t}}\right\}$ (where $v_{i}^{(r)} v_{j}$ means that there are $r$ edges between $v_{i}$ and $v_{j}$ ) is a realization of $\pi$ such that $\mathrm{d}_{\mathrm{G}}\left(v_{\mathrm{i}}\right)=\mathrm{d}_{\mathrm{i}}$ for $1 \leq \mathrm{i} \leq \mathrm{n}, \mathrm{G}$ contains $\mathrm{S}_{\mathrm{L}, \mathrm{M}}^{(\mathrm{r})}$ on $v_{1}, \ldots, v_{\mathrm{L}+\mathrm{M}}$ with $\mathrm{V}^{(\mathrm{r})}\left(\mathrm{K}_{\mathrm{L}}\right)=\left\{v_{1}, \ldots, v_{\mathrm{L}}\right\}$ and $\mathrm{V}\left(\overline{\mathrm{K}}_{\mathrm{M}}^{(\mathrm{r})}\right)=\left\{v_{\mathrm{L}+1}, \ldots, v_{\mathrm{L}+\mathrm{M}}\right\}$ and $\mathrm{G}$ has the number of vertices adjacent to $v_{1}$ in $\left\{v_{\mathrm{L}+\mathrm{M}+1}, \ldots, v_{\mathrm{d}_{1}+1}\right\}$ larger than that of $\mathrm{H}$. This contradicts the choice of $\mathrm{H}$. Repeating this procedure, we can see that $\pi_{i}$ is potentially $A_{L-i}^{(r)}$-graphic successively for $i=2, \ldots, L$. In particular, $\pi_{L}$ is r-graphic.

Conversely, suppose that $\pi_{\mathrm{L}}$ is $r$-graphic and is realized by a graph $\mathrm{G}_{\mathrm{L}}$ with a vertex set $V\left(G_{L}\right)=\left\{v_{L+1}, \ldots, v_{n}\right\}$ such that $d_{G_{L}}\left(v_{i}\right)=d_{i}$ for $L+1 \leq$ $i \leq n$. For $i=L, L-1 \ldots, 1$ form $G_{i-1}$ from $G_{i}$ by adding a new vertex $v_{i}$ that is adjacent to each of $v_{i+1}, \ldots, v_{\mathrm{L}+\mathrm{M}}$ with $r$-edges and also to the vertices of $G_{i}$ with degrees $s_{L+M+1}^{i-1}-r, \ldots, d_{d_{i}+1}^{i-1}-r$. Then for each $i, G_{i}$ has degrees given by $\pi_{i}$ and $G_{i}$ contains $S_{L-i, M}^{(r)}$ on $L+M-i$ vertices $v_{i+1}, \ldots, v_{L+M}$ whose degrees are $\mathrm{d}_{\mathrm{i}+1}-\mathrm{ir}, \ldots, \mathrm{d}_{\mathrm{L}+\mathrm{M}}-\mathrm{ir}$ so that $\mathrm{V}\left(\mathrm{K}_{\mathrm{L}-\mathrm{i}}^{(\mathrm{r})}\right)=\left\{v_{\mathrm{i}+1}, \ldots, v_{\mathrm{L}}\right\}$ and $\mathrm{V}\left(\overline{\mathrm{K}}_{\mathrm{M}}^{(\mathrm{r})}\right)=\left\{v_{\mathrm{L}+1}, \ldots, v_{\mathrm{L}+\mathrm{M}}\right\}$. In particular, $\mathrm{G}_{0}$ has degrees given by $\pi$ and contains $S_{L, M}^{(r)}$ on $L+M$ vertices $v_{1}, \ldots, v_{L+M}$ whose degrees are $d_{1}, \ldots, d_{L+M}$ so that $\mathrm{V}\left(\mathrm{K}_{\mathrm{L}}^{(\mathrm{r})}\right)=\left\{v_{1}, \ldots, v_{\mathrm{L}}\right\}$ and $\mathrm{V}\left(\overline{\mathrm{K}}_{\mathrm{M}}^{(\mathrm{r})}\right)=\left\{v_{\mathrm{L}+1}, \ldots, v_{\mathrm{L}+\mathrm{M}}\right\}$. Hence the result follows.

The following is a sufficient condition for an r-graphic sequence to be potentially $A_{L, M}^{(r)}$-graphic.

Theorem 19 Let $\mathrm{n} \geq \mathrm{L}+\mathrm{M}$ and let $\pi=\left(\mathrm{d}_{1}, \ldots, \mathrm{d}_{\mathrm{n}}\right)$ be an $\mathrm{r}$-graphic sequence. If $\mathrm{d}_{\mathrm{L}+\mathrm{M}} \geq 2 \mathrm{rL}+\mathrm{rM}-2$, then $\pi$ is potentially $\mathrm{A}_{\mathrm{L}, \mathrm{M}}^{(\mathrm{r})}$-graphic.

Proof. Let $n \geq L+M$ and let $\pi=\left(d_{1}, \ldots, d_{n}\right)$ be a non-increasing $r$ graphic sequence with $d_{L+M} \geq 2 r L+r M-2$. By using the argument similar 
to Theorem $8, \pi$ is potentially $K_{L}^{(r)}$-graphic and hence by Lemma $17, A_{L}^{(r)}$ graphic. Therefore, we assume that $\mathrm{G}$ is a realization of $\pi$ with a vertex set $\mathrm{V}(\mathrm{G})=\left(v_{1}, \ldots, v_{\mathrm{n}}\right)$ such that $\mathrm{d}_{\mathrm{G}}\left(v_{\mathrm{i}}\right)=\mathrm{d}_{\mathrm{i}},(1 \leq \mathrm{i} \leq \mathrm{n})$ and $\mathrm{G}$ contains $\mathrm{K}_{\mathrm{L}}^{(\mathrm{r})}$ on $\left(v_{1}, \ldots, v_{\mathrm{L}}\right)$, that is, $\mathrm{V}\left(\mathrm{K}_{\mathrm{L}}^{(\mathrm{r})}\right)=\left\{v_{1}, \ldots, v_{\mathrm{L}}\right\}$ and

$$
\mathrm{t}=\mathrm{e}_{\mathrm{G}}\left(\left\{v_{1}, \ldots, v_{\mathrm{r}_{1}}, \ldots, v_{\mathrm{L}}\right\},\left\{v_{\mathrm{L}+1}, \ldots, v_{\mathrm{L}+\mathrm{s}_{1}}, \ldots, v_{\mathrm{L}+\mathrm{M}}\right\}\right)
$$

(that is, the number of edges between $\left\{v_{1}, \ldots, v_{\mathrm{L}}\right\}$ and $\left\{v_{\mathrm{L}+1}, \ldots, v_{\mathrm{L}+\mathrm{M}}\right\}$ ) is maximum. If $t=r L M+r s_{1} s_{2}+s_{j} \sum_{i=1}^{j-1} r s_{i}$, for $j=3,4, \ldots, p$, then the cardinality of the edge set of $S_{L, M}^{(r)}$ is same as $t$ and therefore $G$ contains $S_{L, M}^{(r)}$ on the vertices $v_{1}, v_{2}, \ldots, v_{\mathrm{L}+\mathrm{M}}$ with $\mathrm{V}^{(\mathrm{r})}\left(\mathrm{K}_{\mathrm{M}}\right)=\left\{v_{1}, v_{2}, \ldots, v_{\mathrm{L}}\right\}$ and

$$
\mathrm{V}\left(\overline{\mathrm{K}^{(\mathrm{r})}} \mathrm{M}\right)=\left\{v_{\mathrm{L}+1}, v_{\mathrm{L}+2}, \ldots, v_{\mathrm{L}+\mathrm{s}_{1}}, \ldots, v_{\mathrm{L}+\mathrm{M}}\right\}
$$

In other-words, $\pi$ is potentially $\bar{A}_{L, M}^{(r)}$-graphic. Assume that $t<\left\{r L M+r s_{1} s_{2}+\right.$ $\left.s_{j} \sum_{i=1}^{j-1} r s_{i}\right\}$, for $j=3,4, \ldots, p$. Then there exists a $v_{k} \in\left\{v_{1}, v_{2}, \ldots, v_{s_{i}}\right\}$ and $v_{m} \in\left\{v_{s_{i}+1}, v_{s_{i}+2}, \ldots, v_{s_{i}+s_{j}}\right\},(i \neq j)$ such that $v_{k}^{r} v_{m} \notin E(G)$. Let

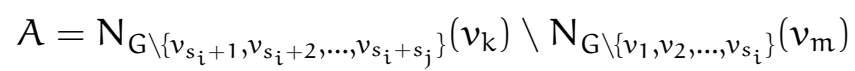

and

$$
\mathrm{B}=\mathrm{N}_{\mathrm{G} \backslash\left\{v_{s_{i}+1}, v_{s_{i}+2}, \ldots, v_{s_{i}+s_{j}}\right\}}\left(v_{\mathrm{k}}\right) \cap \mathrm{N}_{\mathrm{G} \backslash\left\{v_{1}, v_{2}, \ldots, v_{s_{i}}\right\}}\left(v_{\mathrm{m}}\right) .
$$

Then $e_{G}(x, y)=r$ for $x \in N_{G \backslash\left\{v_{1}, \ldots, v_{L}\right\}}\left(v_{m}\right)$ and $y \in N_{G \backslash\left\{v_{1}, \ldots, \nu_{L+M}\right\}}\left(v_{k}\right)$. Otherwise, if $e_{G}(x, y)<r$, then $G^{\prime}=\left(G \backslash\left\{v^{(r)} y, v_{m}^{(r)} x\right\}\right) \cup\left\{v_{k}^{(r)} v_{m}, x^{(r)} y\right\}$ is a realization of $\pi$ and contains $\bar{S}_{\mathrm{L}, M}^{(\mathrm{r})}$ on $v_{1}, \ldots, v_{\mathrm{L}+\mathrm{M}}$ with $\mathrm{V}\left(\mathrm{K}_{\mathrm{L}}^{(\mathrm{r})}\right)=\left\{v_{1}, \ldots, v_{\mathrm{L}}\right\}$ and $\left(\overline{\mathrm{K}}_{\mathrm{M}}^{(\mathrm{r})}\right)=\left\{v_{\mathrm{L}+1}, \ldots, v_{\mathrm{L}+\mathrm{M}}\right\}$ such that

$$
e_{\mathrm{G}^{\prime}}\left(\left\{v_{1}, \ldots, v_{\mathrm{L}}\right\},\left\{v_{\mathrm{L}+1}, \ldots, v_{\mathrm{L}+\mathrm{M}}\right\}\right)>t,
$$

which contradicts the choice of G. Thus B is r-complete. We consider the following cases.

Let $A=\emptyset$. Then $2 r L+r M-2 \leq d_{k}=d_{G}\left(v_{k}\right)<r L+r M-1+r|B|$, and so $|B| \geq r L$. Since each vertex in $N_{G} \backslash v_{1}, \ldots, v_{L}\left(v_{m}\right)$ is adjacent to each vertex in $B$ by $r$ edges and $\left|N_{G \backslash\left\{v_{1}, \ldots, \nu_{L}\right\}}\left(v_{m}\right)\right| \geq 2 r L+r M-2=r L+r M-1$. It can be easily seen that the $r$ induced subgraph of $\mathrm{N}_{\mathrm{G} \backslash\left\{v_{1}, \ldots, v_{\mathrm{L}}\right\}}\left(v_{\mathrm{m}}\right) \cup\left\{v_{\mathrm{m}}\right\}$ in $\mathrm{G}$ contains $\overline{\mathrm{S}}_{\mathrm{L}, \mathrm{M}}^{(\mathrm{r})}$ 
as a subgraph. Thus $\pi$ is potentially $\bar{A}_{\mathrm{L}, M^{-}}^{(r)}$ graphic.

Let $A \neq \emptyset$. Let $a \in A$. If there are $x, y \in N_{G \backslash\left\{v_{1}, \ldots, v_{L}\right\}}\left(v_{m}\right)$ such that $e_{G}(x, y)<r$ then $G^{\prime}=\left(G \backslash\left\{v_{m}^{(r)} x, v_{m}^{(r)} y, v_{k}^{(r)} a\right\}\right) \cup\left\{v_{k}^{(r)} v_{m}, a^{(r)} v_{m}, x^{(r)} y\right\}$ is a realization of $\pi$ and contains $\bar{S}_{\mathrm{L}, M}^{(\mathrm{r})}$ on $v_{1}, \ldots, v_{\mathrm{L}+\mathrm{M}}$ with $\mathrm{V}\left(\mathrm{K}_{\mathrm{L}}^{(\mathrm{r})}\right)=\left\{v_{1}, \ldots, v_{\mathrm{L}}\right\}$ and $\mathrm{V}\left(\overline{\mathrm{K}}_{\mathrm{M}}^{(\mathrm{r})}\right)=\left\{v_{\mathrm{L}+1}, \ldots, v_{\mathrm{L}+\mathrm{M}}\right\}$ such that $\mathrm{e}_{\mathrm{G}^{\prime}}\left(\left\{v_{1}, \ldots, v_{\mathrm{L}}\right\},\left\{v_{\mathrm{L}+1}, \ldots, v_{\mathrm{L}+\mathrm{M}}\right\}\right)>\mathrm{t}$ which contradicts the choice of $G$. Thus $N_{G \backslash\left\{v_{1}, \ldots, v_{L}\right\}}\left(v_{m}\right)$ is r-complete. Since

$$
\left|\mathrm{N}_{\mathrm{G} \backslash\left\{v_{1}, \ldots, \nu_{\mathrm{L}}\right\}}\left(v_{\mathrm{m}}\right)\right| \geq \mathrm{rL}+\mathrm{rM}-1 \text { and } \mathrm{e}_{\mathrm{G}}\left(v_{\mathrm{m}}, z\right)=\mathrm{r},
$$

for any $z \in N_{G \backslash\left\{v_{1}, \ldots, v_{L}\right\}}\left(v_{m}\right)$, it is easy to see that the induced r-subgraph of $\mathrm{N}_{\mathrm{G} \backslash\left\{v_{1}, \ldots, v_{\mathrm{L}}\right\}}\left(v_{\mathrm{m}}\right) \cup\left\{v_{\mathrm{m}}\right\}$ in $\mathrm{G}$ is r-complete, and so contains $\overline{\mathrm{S}}_{\mathrm{L}, \mathrm{M}}^{(\mathrm{r})}$ as a r-subgraph. Thus $\pi$ is potentially $\bar{A}_{L, M}^{(r)}$-graphic.

Theorem 20 If $\pi=\left(\mathrm{d}_{1}, \mathrm{~d}_{2}, \ldots, \mathrm{d}_{\mathrm{n}}\right)$ is an $\mathrm{r}$-graphic sequence such that $\sigma(\pi)$ is at least $\left(\mathrm{n}^{2}-3 \mathrm{n}+8\right) \mathrm{r}$, then $\pi$ is potentially $\mathrm{K}_{4}^{(\mathrm{r})}$-graphic.

Proof. Let $\pi=\left(d_{1}, d_{2}, \ldots, d_{n}\right)$ be an $r$-graphic sequence such that $d_{1} \geq d_{2} \geq$ $\ldots \geq d_{n} \geq 1$ and $\sigma(\pi)=\left(n^{2}-3 n+8\right) r$. Suppose $G$ is a graphical realization of $\pi$ and $e(G)$ is the size of $G$. Then $2 e(G)=\sigma(\pi)$ and $2 e\left(G^{c}\right)=n b(n-1)-$ $\sigma(\pi)=n r(n-1)-\left(n^{2}-3 n+6\right) r=r(2 n-6)$, so that $e\left(G^{c}\right)=r(n-3)$, where $\mathrm{G}^{\mathrm{c}}$ is the complement of the $\mathrm{r}$-graph $\mathrm{G}$. An extremal problem is $r$-graph $\mathrm{G}$ is obtained by deleting $r(n-3)$ independent edges from the complete r-graph $K_{n}^{(r)}$ of order $n$. Hence the largest vertex number of independent sets in $G^{c}$ is 3. This implies that the largest possible complete $r$-subgraph of $G$ is of order 3. As $1 \leq n-3 \leq 3$. Hence there is no complete $r$-subgraph of order 4 in $G$. Therefore, we have

$$
\sigma\left(K_{4}^{(r)}, n\right) \geq\left(n^{2}-3 n+6\right) r+2 r=\left(n^{2}-3 n+8\right) r
$$

Now Suppose that $\pi=\left(d_{1}, d_{2}, \ldots, d_{n}\right)$ is r-graphic sequence with $d_{1} \geq d_{2} \geq$ $\ldots \geq d_{n} \geq r$ and $\sigma(\pi) \geq\left(n^{2}-3 n+8\right) r$. Then every graphical realization $\mathrm{G}$ of $\pi$ is obtained by removing at most $r(n-4)$ edges from the r-complete graph $K_{n}^{(r)}$. Hence the maximal complete subgraph of $G$ has order at least $n-(n-4)=4$. Thus $G$ is potentially $K_{4}^{(r)}$. In other words,

$$
\sigma\left(K_{4}^{(r)}, n\right) \leq\left(n^{2}-3 n+8\right) r
$$

Combining (3) and (4), the result follows.

Acknowledgements. The authors thank the anonymous referee for his useful comments and suggestions. 


\section{References}

[1] D. de Caen, An upper bound on the sum of squares of degrees in a graph, Discrete Math. 185 (1998) 245-248. $\Rightarrow 38$

[2] V. Chungphaisan, Conditions for a sequences to be r-graphic, Discrete Math. 7 (1974) 31-39. $\Rightarrow 36,37$

[3] P. Erdős, T. Gallai, Graphs with prescribed degrees (in Hungarian) Matematikai Lapok 11(1960) 264-274. $\Rightarrow 36$

[4] P. Erdős, M. S. Jacobson, J. Lehel, Graphs realizing the same degree sequences and their respective clique numbers, in: Graph Theory, Combinatorics and Applications, vol. 1, John Wiley and Sons, New York, 1991, 439-449. $\Rightarrow 37$

[5] D. R. Fulkerson, A. J. Hoffman, M. H. McAndrew, Some properties of graphs with multiple edges, Canad. J. Math. 17 (1965) 166-177. $\Rightarrow 36$

[6] D. J. Kleitman, D. L. Wang, Algorithm for constructing graphs and digraphs with given valencies and factors, Discrete Math. 6 (1973) 79-88. $\Rightarrow 36$

[7] J. S. Li, Z. X. Song, R. Luo, The Erdős-Jacobson-Lehel conjecture on potentially $p_{k}$-graphic sequences is true, Sci. China Ser. A 41 (1998) 510-520. $\Rightarrow 37$

[8] J. S. Li, Z. X. Song, An extremal problem on the potentially $p_{k}$-graphic sequence, Discrete Math. 212 (2000) 223-231. $\Rightarrow 37$

[9] S. Pirzada, An Introduction to Graph Theory, Universities Press, Orient Blakswan, Hyderabad, India 2012. $\Rightarrow 36$

[10] S. Pirzada, B. A. Chat, Potentially graphic sequences of split graphs, Kragujevac J. Math. 38, 1 (2014) 73-81. $\Rightarrow 37,38$

[11] S. Pirzada, B. A. Chat, F. A. Dar, Graphical sequences of some family of induced subgraphs, J. Algebra Comb. Discrete Appl. 2(2) (2015) 95-109. $\Rightarrow 38$

[12] A. R. Rao, The clique number of a graph with a given degree sequence, Proc. Symposium on Graph Theory (ed. A. R. Rao, Macmillan and Co. India Ltd, I.S.I. Lecture Notes Series, 4 (1979) 251-267. $\Rightarrow 37$

[13] J. H. Yin, Conditions for $r$-graphic sequences to be potentially $\mathrm{K}_{\mathrm{m}+1}^{\mathrm{r}}$-graphic, Discrete Math. 309 (2009) 6271-6276. $\Rightarrow 37$

[14] J. H. Yin, A Havel-Hakimi type procedure and a sufficient condition for a sequence to be potentially $\mathrm{S}_{\mathrm{r}, \mathrm{s}}$-graphic, Czechoslovak Math. J. 62,3 (2012) 863867. $\Rightarrow 37,38$ 